\title{
Unintentional sulfonylurea toxicity due to a drug-drug interaction: a case report
}

\author{
Keith Gunaratne ${ }^{1}$, Emily Austin ${ }^{1,2,3}$ and Peter E. Wu 1,3,4* $^{1 *}$
}

\begin{abstract}
Background: Sulfonylureas are widely used for type 2 diabetes mellitus, but these medications carry a risk of hypoglycemia. Drug-drug interactions that inhibit sulfonylurea metabolism and thus increase systemic exposure can cause unintentional sulfonylurea toxicity.

Case presentation: A 56-year-old man presented with severe, recurrent hypoglycemia. He had a history of type 2 diabetes mellitus and was taking the sulfonylurea gliclazide with no prior episodes of hypoglycemia. The onset of his hypoglycemia occurred within days after starting voriconazole and subsequently fluconazole for a fungal pneumonia. Unintentional sulfonylurea toxicity developed due to an adverse drug-drug interaction between gliclazide and these antifungals. Azole antifungals inhibit the metabolism of sulfonylureas resulting in increased systemic exposure and consequent toxicity. After the diagnosis of sulfonylurea toxicity was recognized, the patient was treated initially with dextrose and then administered octreotide to prevent recurrent hypoglycemia. He was successfully managed, his hypoglycemic episodes resolved, and his medications were adjusted to avoid any further adverse interactions.
\end{abstract}

Conclusions: Adverse drug-drug interactions continue to pose challenges to clinicians. Both individual vigilance and system wide strategies are needed to prevent and mitigate consequences. This case highlights an important drug-drug interaction and reviews the presentation, management and antidotal therapy of sulfonylurea toxicity.

Keywords: Education, Toxicology, Pharmacology, Drug-interaction

\section{Background}

Sulfonylureas are commonly used for type 2 diabetes mellitus. These compounds stimulate glucose-independent endogenous insulin release [1] and carry an inherent risk of hypoglycemia, particularly in the setting of intentional overdose or due to adverse drug interactions. Sulfonylureas are extensively metabolized by the hepatic cytochrome P450 (CYP) 2C9 isoform and medications that inhibit this enzyme can result in impaired sulfonylurea metabolism, increased exposure and consequent toxicity [2].

We present a case of severe, recurrent hypoglycemia in a patient taking the sulfonylurea gliclazide who was prescribed voriconazole followed by fluconazole, two inhibitors of the hepatic CYP2C9 enzyme. Once recognized,

\footnotetext{
*Correspondence: peter.wu@uhn.ca

${ }^{4}$ Division of General Internal Medicine, University Health Network, 200 Elizabeth St. 14EN206, Toronto, ON M5G 2C4, Canada

Full list of author information is available at the end of the article
}

this unintentional overdose was successfully managed initially with dextrose, and then octreotide.

\section{Case presentation}

A 56-year-old man presented to the emergency department with new onset hypoglycemia. He had a history of type 2 diabetes treated with metformin $(2500 \mathrm{mg}$ orally daily in divided doses), gliclazide modified release $(90 \mathrm{mg}$ orally daily), and a multiple daily injection insulin regimen (insulin glargine 18 units subcutaneously at night and insulin lispro 6 units subcutaneously with meals). He was adherent with all medications and had never developed hypoglycemia. Past medical history was notable for a bone marrow transplantation for myelofibrosis 8 months prior and long-term treatment with prednisone (5 mg orally daily), resulting in an immunocompromised state.

One week before presentation, he was evaluated in clinic for persistent cough. Findings on a Computed Tomography scan of the thorax were suspicious for 
fungal pneumonia, and he was empirically treated with voriconazole ( $200 \mathrm{mg}$ orally twice daily). He was reassessed in clinic 6 days later reporting lethargy, visual disturbances, and hallucinations. Voriconazole intolerance was suspected, as visual disturbances and hallucinations are well described side effects [3], and his antifungal was switched to fluconazole (480 mg orally daily). Incidentally, after starting antifungal therapy, he had not required any short-acting insulin due to glucose readings of $54-72 \mathrm{mg} / \mathrm{dL}(3.0-4.0 \mathrm{mmol} / \mathrm{L}$ ) (reference range $72-126 \mathrm{mg} / \mathrm{dL} ; 4.0-7.0 \mathrm{mmol} / \mathrm{L})$.

The day after initiating fluconazole, he presented to a follow-up appointment at $1800 \mathrm{~h}$ weak and tremulous. His capillary glucose was $31 \mathrm{mg} / \mathrm{dL}(1.7 \mathrm{mmol} / \mathrm{L})$. He was given orange juice, felt better, and sent to the emergency department, where a repeat measurement at $2000 \mathrm{~h}$ was $34 \mathrm{mg} / \mathrm{dL}(1.9 \mathrm{mmol} / \mathrm{L})$. He consumed more juice and a sandwich, his symptoms improved and subsequent hourly glucose measurements ranged between 68 and $106 \mathrm{mg} / \mathrm{dL}(3.8-5.9 \mathrm{mmol} / \mathrm{L})$. At $0100 \mathrm{~h}$ his glucose was $106 \mathrm{mg} / \mathrm{dL}(5.9 \mathrm{mmol} / \mathrm{L})$ and he fell asleep.

At $0430 \mathrm{~h}$, he was woken for reassessment. He was confused, lethargic and glucose was $25 \mathrm{mg} / \mathrm{dL}(1.4 \mathrm{mmol} / \mathrm{L})$. The recurrent nature of his hypoglycemia in the setting of recent azole antifungal co-prescription was concerning for gliclazide toxicity due to an adverse drug interaction.

He was treated with a $50 \mathrm{cc}$ bolus of $50 \%$ dextrose followed by a $100 \mathrm{cc}$ per hour infusion of $10 \%$ dextrose. In consultation with the regional poison centre, he was given octreotide $75 \mu \mathrm{g}$ subcutaneously for suspected sulfonylurea toxicity and the intravenous dextrose was discontinued as his blood sugar normalized. One further dose of octreotide was administered 6-h later and his hypoglycemic episodes abated with no recurrence during hospitalization. His gliclazide was held and his medical records were updated to document this interaction.

\section{Discussion and conclusions}

Sulfonylureas stimulate insulin release independent of plasma glucose concentration, thereby creating a risk for hypoglycemia. Hypoglycemia can occur due to accidental ingestion, intentional overdose [4], or as a result of adverse drug-drug interactions [5]. Sulfonylureas are metabolized by CYP2C 9 and the most common drug-drug interaction causing toxicity is inhibition of this enzyme [2]. The azole antifungals voriconazole and fluconazole are examples of CYP2C9 inhibitors and there are many commonly prescribed drugs that can inhibit or induce this enzyme (Table 1$)[1,2,5,6]$. One retrospective study of 3884 patients on sulfonylureas revealed that $20 \%$ were co-prescribed a CYP2C9 inhibitor and were more likely $(12.8 \%$ vs. $8.9 \%)$ to have a fasting glucose below $72 \mathrm{mg} / \mathrm{dL}$ (4 mmol/L) [5]. Another retrospective population-based study of 909 elderly patients on sulfonylureas admitted to hospital with hypoglycemia were more than six times as likely to have been prescribed cotrimoxazole (a CYP2C9 inhibitor) in the previous week than matched controls [7].

The management goals of sulfonylurea toxicity are to restore and maintain euglycemia. The initial hypoglycemic episode will require an intravenous (IV) dextrose bolus, followed by complex carbohydrates; however, ongoing IV dextrose use can complicate recovery and should be avoided. Glucose independently stimulates insulin release, thus ongoing administration of dextrose in the setting of sulfonylurea toxicity will perpetuate a dangerous cycle of recurrent hypoglycemia [8]. Octreotide is a long-acting somatostatin analog that prevents the secretion of insulin downstream of sulfonylurea action. It is recommended as part of sulfonylurea toxicity management to prevent recurrent hypoglycemia $[8,9]$. A prospective randomized study of 40 emergency department patients with sulfonylurea-induced hypoglycemia demonstrated higher glucose levels and fewer episodes of recurrent hypoglycemia over an 8-h period after treatment with octreotide $75 \mu \mathrm{g}$ subcutaneously compared to placebo [10].

There is limited published data to guide dosing protocols for octreotide. A recommended strategy is octreotide $50-100 \mu \mathrm{g}$ subcutaneously every $6-12 \mathrm{~h}$ as needed [8]. Patients require observation for a minimum of $12-\mathrm{h}$ after the last octreotide dose to ensure no recurrence of hypoglycemia [8]. Octreotide is well tolerated and only minor adverse events have been associated with its use [4].

This case also highlights the importance of vigilance for drug-drug interactions, and the need for better systems to aid in identifying clinically significant

Table 1 Common medications that can interact with sulfonylureas $[1,2,5,6]$

\begin{tabular}{|c|c|c|}
\hline Drug & Mechanism & Effect \\
\hline $\begin{array}{l}\text { Fluconazole, ketoconazole, voriconazole, amiodarone, co-trimoxazole (sulfamethoxazole } \\
\text { component), fluoxetine, fluvoxamine, metronidazole }\end{array}$ & Inhibition of CYP2C9 & Hypoglycemia \\
\hline Carbamazepine, phenobarbital, rifampin, St. John's wort, dexamethasone & Induction of CYP2C9 & $\begin{array}{l}\text { Reduced efficacy } \\
\text { (hyperglyce- } \\
\text { mia) }\end{array}$ \\
\hline
\end{tabular}


interactions. Drug-drug interactions are innumerable and it is unreasonable to expect clinicians to recall every potential interaction. However, whenever new medications are started it is sensible for clinicians to search for interactions between the new drug and any high-risk medications with narrow therapeutic indices (e.g. oral antihyperglycemics, anticoagulants, opioids). Collaboration with pharmacists and iterative medication review would assist in identifying adverse drug interactions.

Sulfonylureas carry an inherent risk of hypoglycemia due to a mechanism of glucose-independent insulin release. Unintentional toxicity can occur if sulfonylurea metabolism is impaired by a co-prescribed medication that inhibits CYP2C9. In this case, the addition of azole antifungals was the culprit. Sulfonylurea toxicity is readily treated if recognized quickly. Management includes judicious use of intravenous dextrose, ingestion of complex carbohydrates and administration of octreotide to prevent recurrent hypoglycemia. Drug-drug interactions remain a common and preventable source of harm associated with medical care, both individual vigilance and system wide changes are necessary to prevent and mitigate these adverse drug events.

\section{Abbreviation}

CYP: cytochrome P450

\section{Authors' contributions}

All authors (KG, EA, PEW) were involved with the design and conception of the case report, drafting and revising of the manuscript, and reviewing the literature. All authors read and approved the final manuscript.

\section{Author details}

${ }^{1}$ Department of Medicine, University of Toronto, Toronto, ON, Canada. ${ }^{2}$ Division of Emergency Medicine, University of Toronto, Toronto, ON, Canada.

${ }^{3}$ Division of Clinical Pharmacology \& Toxicology, University of Toronto, Toronto, ON, Canada. ${ }^{4}$ Division of General Internal Medicine, University Health Network, 200 Elizabeth St. 14EN206, Toronto, ON M5G 2C4, Canada.

\section{Acknowledgements}

We thank Dr. Margaret Thompson, Medical Director of the Ontario Poison Centre, and Dr. Irfan Dhalla, Associate Professor of Medicine and General Internal Medicine specialist at the University of Toronto for comments made on an earlier version of this manuscript.

\section{Competing interests}

The authors declare that they have no competing interests.
Availability of data and materials Not applicable.

\section{Consent for publication}

Written patient consent has been obtained.

Ethics approval and consent to participate

Not applicable.

\section{Funding}

No funding was received for this case report.

\section{Publisher's Note}

Springer Nature remains neutral with regard to jurisdictional claims in published maps and institutional affiliations.

Received: 18 January 2018 Accepted: 7 May 2018

Published online: 21 May 2018

\section{References}

1. Harrigan RA, Nathan MS, Beattie P. Oral agents for the treatment of type 2 diabetes mellitus: pharmacology, toxicity, and treatment. Ann Emerg Med. 2001;38:68-78.

2. Holstein A, Beil W, Kovacs P. CYP2C metabolism of oral antidiabetic drugsimpact on pharmacokinetics, drug interactions and pharmacogenetic aspects. Expert Opin Drug Metab Toxicol. 2012;8:1549-63.

3. Levine MT, Chandrasekar PH. Adverse effects of voriconazole: over a decade of use. Clin Transplant. 2016;30:1377-86.

4. Glatstein M, Scolnik D, Bentur Y. Octreotide for the treatment of sulfonylurea poisoning. Clin Toxicol. 2012;50:795-804.

5. Tirkkonen T, Heikkilä P, Huupponen R, Laine K. Potential CYP2C9-mediated drug-drug interactions in hospitalized type 2 diabetes mellitus patients treated with the sulphonylureas glibenclamide, glimepiride or glipizide. J Intern Med. 2010;268:359-66.

6. May M, Schindler C. Clinically and pharmacologically relevant interactions of antidiabetic drugs. Ther Adv Endocrinol Metab. 2016;7:69-83.

7. Juurlink DN, Mamdani M, Kopp A, Laupacis A, Redelmeier DA. Drug-drug interactions among elderly patients hospitalized for drug toxicity. JAMA. 2003;289:1652.

8. Klein-Schwartz W, Stassinos GL, Isbister GK. Treatment of sulfonylurea and insulin overdose. Br J Clin Pharmacol. 2016;81:496-504.

9. McLaughlin SA, Crandall CS, McKinney PE, Litovitz T, Klein-Schwartz W, Dyer K, et al. Octreotide: an antidote for sulfonylurea-induced hypoglycemia. Ann Emerg Med. 2000;36:133-8.

10. Francis M, Langhan T, Prosser J, Hoffman R, Fasano CJ, O'Malley G, et al. Comparison of octreotide and standard therapy versus standard therapy alone for the treatment of sulfonylurea-induced hypoglycemia. Ann Emerg Med. 2008;51:795-6. 